\title{
Estudio fractal de la superficie de la hoja de la especie vegetal Copaifera sp. haciendo uso del Microscopio de Fuerza Atómica-AFM
}

\author{
Study fractal leaf surface of the plant species Copaifera sp. using the Microscope \\ Atomic-Force-AFM
}

Mario Omar Calla Salcedo, Robert Ronald Maguiña Zamora, y José Carlos Tavares Carvalho

\begin{abstract}
Universidade Federal de Amapá, Rodovia Juscelino Kubitschek de Oliveira, Km 02 - s/n, Bairro Jardim Marco Zero - Macapá -AP, CEP 68.902-280
\end{abstract}

DOI: https://doi.org/10.33017/RevECIPeru2016.0002/

\section{Resumen}

Las especies de copaifera sp, que también son denominadas de copaíba y que son ampliamente utilizadas en la medicina popular debido a sus propiedades etnofarmacológicas. Este trabajo fue realizado con el objetivo de padronizar las hojas, mediante el estudio de la textura superficial da la hoja, para eso se necesita la obtención de los parámetros fractales como la dimensión Fractal, Lagunaridad y succolaridad, haciendo uso de los datos que proporciona el Microscopio de Fuerza Atómica, más conocido como AFM (por las siglas en inglés) se trabajó con la área óptima $\left(25 \times 25 \mu \mathrm{m}^{2}\right)$, con el procesamiento de datos y aplicando la geometría fractal, se desarrollaron los algoritmos haciendo uso del programa computacional Fortran 77, el estudio fue realizado a partir de la dificultad que se tiene al diferenciar una especie de otra de la Copaifera $s p$, ya que para hacer tal identificación se necesita la flor y hoja, esto es porque la planta solo florece una vez al año, y por eso se está proponiendo una manera más fácil, y efectiva da tal identificación solo haciendo uso de la hoja de la Copaifera sp, para el cálculo de la dimensión fractal se hizo uso del método de conteo de cajas (Box-Counting), se usó este método por su simplicidad y exactitud, la dimensión fractal va a servir para calcular la rugosidad y porosidad de la superficie de la hoja de la Copaifera sp., donde el valor de la rugosidad obtenido por medio de la dimensión fractal es más exacto que el cálculo de la rugosidad por medio de la geometría Euclidiana. La lagunaridad, es otro parámetro fractal, que sirve para medir el grado de uniformidad de los huecos en la superficie de la hoja de la Copaifera $s p$, para el cálculo de la lagunaridad se hizo uso de método conteo de Caja Diferencial (Differential Box Counting) que es un método basado en el conteo de cajá (Box-Counting), si la lagunaridad es mucho mayor que 1, existe mayor desorden de los huecos, si la lagunaridad es más próximo a 1, existe menor desorden, ahora si la lagunaridad es igual 1, la superficie es completamente uniforme, seria invariante a la rotación. La succolaridad es el último parámetro fractal que se aplicó al estudio de la superficie de la hoja, que mide la capacidad de un flujo de agua de atravesar toda la superfície en una determinada dirección, a este proceso se le llama percolación, se midió la succolaridad en las cuatro direcciones es decir de arriba hacia abajo, de abajo hacia arriba, de izquierda a la derecha, y por ultimo de derecha a la izquierda. Teniendo calculado los tres parámetros fractales: dimensión fractal, lagunaridad, y succolaridad, se tiene caracterizado completamente la superficie foliar.

Descriptores: Copaifera. Dimensión Fractal. Lagunaridad. Succolaridad. Textura.

\begin{abstract}
The species of Copaifera sp. which are also called copal are widely used in folk medicine due to its ethnopharmacological properties. This work was accomplished with the purpose of the possibility of standardization of the leaves, on the study of the surface texture of the leaf, for this you need to obtain the fractal parameters as fractal dimension (roughness, porosity), lacunarity (rotational invariance of the holes ) and succolarity (percolation), making use of the data of the Atomic Force Microscopy (AFM) worked with the
\end{abstract}


optimal area $\left(25 \times 25 \mu \mathrm{m}^{2}\right)$, with the data process and applying fractal mathematics, algorithms were developed with the computer program Fortran 77 . The study was conducted from difficulty that one has to distinguish one species from another of Copaifera sp., and to make such identification is needed flower and leaf Copaifera sp., this is because the plant blooms only once a year. That's why it is proposing an easier and effective way to such identification, only making use of leaf Copaifera $s p$. for the calculation of the fractal dimension. It will make use of Box Counting method for its simplicity and exactitude, which will serve to calculate the roughness and porosity of the surface of the sheet Copaifera sp. It is expected that the value of roughness obtained by the Fractal geometry is more accurate, the calculation of roughness with Euclidean mathematics. The Lacunarity is another fractal parameter used to determine readily the uniformity of the holes for the calculation of lacunarity be made using the method of the counting boxes (Differential Box Counting) which is a method based on the counting boxes (Box-Counting), but the lacunarity is much greater than one, there is greater disorder of the holes. The lacunarity is closer to 1, there is less clutter, now the lacunarity is equal to 1, the surface is completely uniform, is down is invariant rotation, it is expected that lacunarity of Copaifera sp leaf is close to an a succolarity is the last fractal parameter that is doing applied to the study of surfaces, which measures the ability of a flow through the entire surface that serves to measure the percolation surface level. It is measured succolarity in the four directions is down from above down, bottom-up, from left to right, and finally from right to left. When it has calculated the three fractal parameters: fractal dimension, lacunarity and succolarity, it is possible to have fully characterized the leaf surface.

\section{Keywords: Copaifera. Fractal Dimension. Lacunarity. Succolarity. Texture}

\section{Introducción}

En la literatura los datos divergen en cuanto al número exacto de especies pertenecientes al género Copaifera, de acuerdo con Mabbeley (1990), este género posee 30 especies. Sin embargo, fueron encontrados 118 registros de nombres específicos y variedades en el banco de datos taxonómicos del Missouri Botanical Garden (www.tropicos.org), de esas 82 especies consideradas validas y 116 en el International Plant Name Index(www.ipni.org), siendo 72 especies válidas. La discordancia de los números exactos en relación las especies del género debe estar ligada a varias sinonimias botánicas asociadas a algunas especies, reforzando la necesidad de estudios más detallados sobre el género y una revalidación de las especies validas [4] El profesional del área taxonómica debe tener un amplio conocimiento y las habilidades, lo que requiere un largo período de formación; $A$ pesar de las características ofrecidas por las flores y los frutos han sido muy útiles, no siempre están disponibles para el estudio; algunas de las características se pierden durante el proceso de desecamiento, como el color, el brillo, etc. y algunas partes de la planta son simplemente no contemplan para su análisis, tales como las hojas, por ejemplo [7]

Este trabajo tratara sobre el análisis y la caracterización por microscopia de fuerza atómica (AFM, sigla en inglés de Atomic Force Microscopy) el cual es posible el acceso directo a la topografía de superficies en crecimiento de la hoja de la Copaifera sp. Con eso, surge la necesidad de desenvolver métodos matemáticos y computacionales que permitan describir las superficies rugosas e asociar sus características morfológicas al proceso de crecimiento de la superficie de las hojas Copaifera sp.

En general, las imágenes de la superficie de hojas tienen formas irregulares y la geometría euclidiana se utiliza con frecuencia para describir esferas, conos, etc, es inadecuada para ciertas topologías que analizan muchos fragmentos (de forma irregular). Por lo tanto, es necesario utilizar una geometría capaz de proporcionar métodos para describir cuantitativamente la forma general de un objeto.

\section{Materiales y métodos}

\subsection{Colecta de material vegetal y análisis microscópico}

Las hojas de la Copaifera sp. se recogen de una planta adulta en la mañana., serán obtenidos de un área del museo Sacaca en la ciudad de Macapá, ubicada en la Avenida 1583 Feliciano Coelho, las hojas se eligen situados en las partes intermedias de la planta que no han sido afectados por agentes patógenos, ya que afectan a la estructura de la hoja. Todas las hojas se lavaron inicialmente con agua corriente, por lo que las impurezas se retiran a través del proceso de auto-limpieza, y se coloca en recipientes que contienen una pequeña cantidad de agua para evitar que se sequen. Se llevan al Laboratorio de Materiales (LABMAT) de Física de la Universidad Federal de Amapá (UNIFAP) para el análisis y caracterización por AFM.

\subsection{Caracterización Microscopio de Fuerza Atómica-AFM}


Las muestras se lavaron de nuevo en corriente de agua y se dejaron en condiciones ambientales durante un cuarto de hora para que posibles gotas de agua que quedan en su superficie se evaporen y no afectan a la calidad de la imagen por AFM. A continuación, las muestras se cortan a $1,5 \mathrm{~cm}^{2}$ área en la región central de las hojas y se llevan a cabo su análisis bajo AFM.

El análisis se llevaron a cabo en condiciones ambientales en una microscopio de fuerza atómica, modelo Nanosurf Easyscan 2 midiendo en modo Tapping, es decir, utilizando un cantilever NCST-50 de Silicio con recubrimiento reflectante de aluminio, con una frecuencia 160 resonancia $\mathrm{kHz}$ y constante elástica de $7,4 \mathrm{~N} / \mathrm{m}$, espesor $28 \mu \mathrm{m}$, longitud 150 $\mu \mathrm{m}$, ancho $26 \mu \mathrm{m}$ opera en el modo de contraste de fase en una proporción de 1 línea de escaneo por 3 segundos.

\section{Fractales}

\subsection{Dimensión fractal}

La palabra fractal fue acuñado en 1975 por el matemático Benoit Mandelbrot. Tomando esta palabra del latín fractus o frangere, lo que sugiere "fragmentada, rota, discontinua e irregular (E RIVERA ; R LÓPEZ; 2011)

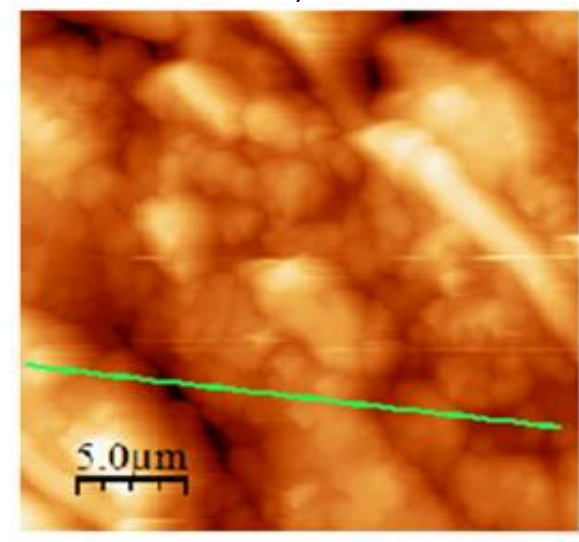

Figura 1: Topografia de la superficie de la Copaifera sp.

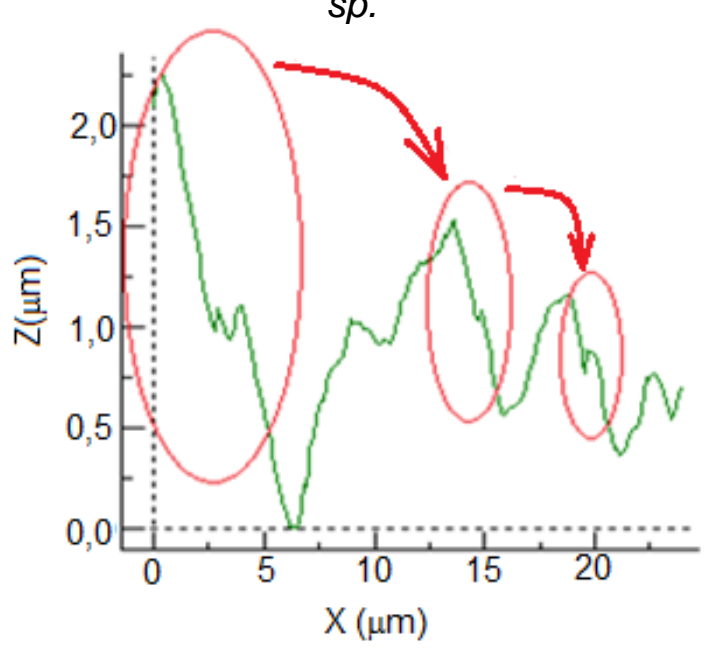

Figura 2: Perfil horizontal de la imagen de la Copaifera sp.

Una de las caracterisiticas generales de sistemas biológicos, donde se incluyen a las superficies de las hojas vegetales es su naturaleza fractal [5]

Se aprecia en la figura 1, la topografia de la hoja de la Copaifera sp. Donde se trazo una linea oblicua donde obtenemos el perfil, y se observa en la figura 2 que existen formas parecidas y cada vez mas pequeña (encerrada con circulos de color rojo). Lo cual de se demuestra que la superficie foliar a nivel nanométrico tiene un comportamiento fractal.

\subsubsection{Método Box-Counting}

El Metodo Box-Counting es el mas conocido, debido a su facilidad de implementacion. Consiste en dividir en mitad la imagen, con cuadrados de tamaños diferentes y contar la cantidad de cuadrados necesarios para cubrir toda la forma presente en la imagen, tal como se representa en la figura 3 [9]

Matemáticamente se calcula la Dimension Fractal $\left(\mathbf{D}_{f}\right)$ de la siguiente manera

$$
D_{f}=\frac{\operatorname{Ln}(n)}{\operatorname{Ln}(N)}
$$

Donde $n$ es el numero de divisiones por lado, y $N$ es el numero de cuadrados que contiene la imagen (cuadrado de color amarillo)
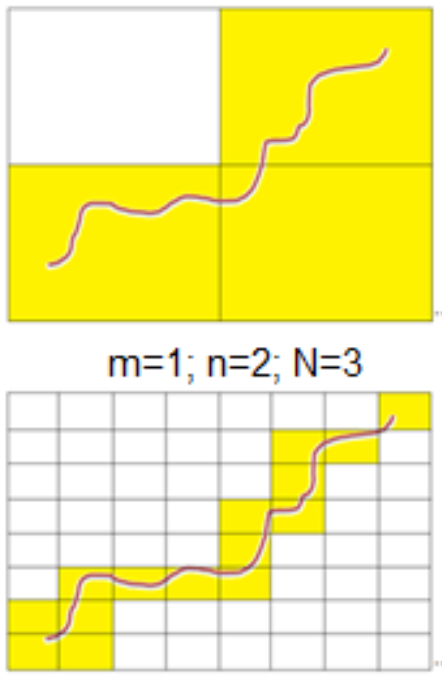

$\mathrm{m}=3 ; \mathrm{n}=8 ; \mathrm{N}=15$

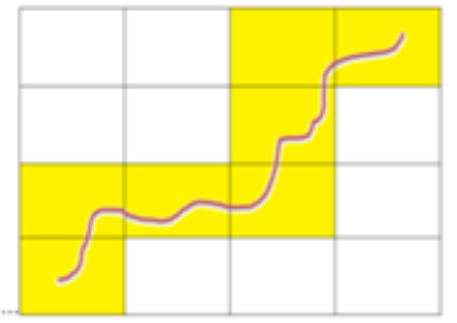

$\mathrm{m}=1 ; \mathrm{n}=4 ; \mathrm{N}=7$

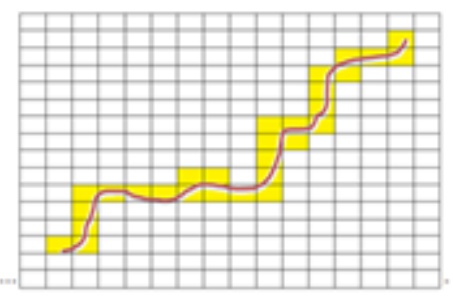

$m=4 ; n=16 ; N=29$
Figura 3: Método Box-Counting 


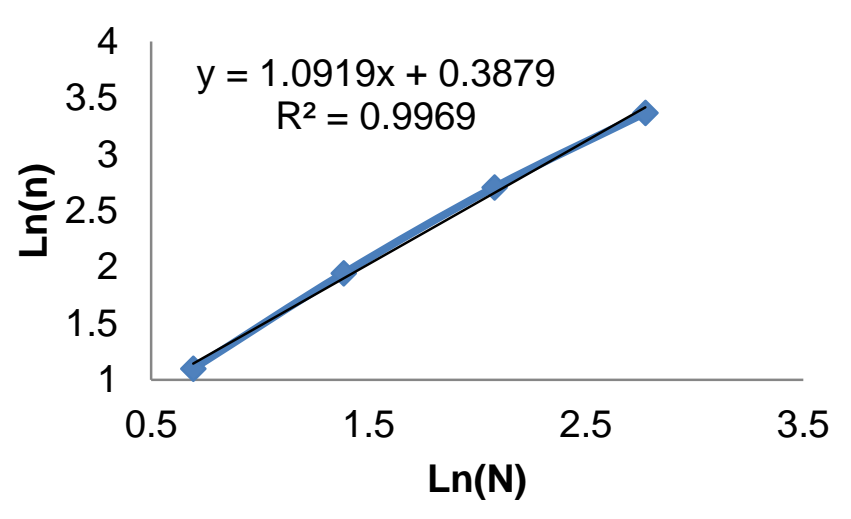

Grafica 1: Dimensión Fractal

$$
D_{f}=\frac{\operatorname{Ln}(n)}{\operatorname{Ln}(N)}=1,0919
$$

Para calcular la rugosidad usando la geometría fractal hacemos uso de la formula (Persson-Tosatti; 2001)

$$
h=h_{a}\left(\frac{\lambda}{\lambda_{a}}\right)^{H}
$$

Donde $h$ es la rugosidad fractal y $\mathrm{H}\left(\mathrm{H}=3-\mathrm{D}_{f}\right)$ se llama parámetro de Hurts,y $h_{a}$ es la amplitud de la rugosidad sobre la escala de longitud $\lambda_{a}$.

Tabla 1: Valores de la rugosidad y dimensión fractal en función del área.

\begin{tabular}{ccc}
\hline AREA $\left(\mu \mathrm{m}^{2}\right)$ & $\begin{array}{c}\text { Dimensión } \\
\text { Fractal }\end{array}$ & $\begin{array}{c}\text { Rugosidad } \\
\text { RMS }(\mu \mathrm{m})\end{array}$ \\
\hline $0,78 \times 0,78$ & 2,1050 & 0,0351 \\
$1,56 \times 1,56$ & 2,1460 & 0,0337 \\
$3,13 \times 3,13$ & 2,1161 & 0,0518 \\
$6,25 \times 6,25$ & 2,1095 & 0,1234 \\
$12,5 \times 12,5$ & 2,1094 & 0,2263 \\
$25 \times 25$ & 2,1263 & 0,4436 \\
$50 \times 50$ & 2,1579 & 0,7026 \\
$100 \times 100$ & 2,2213 & 1,1205
\end{tabular}

Los valores de la dimensión fractal fueron obtenidos por medio del software Gwyddion que es software libre y de código abierto cubierto por la Licencia Pública General de GNU. El cual hace uso del método Box-Counting.

Los valores de la rugosidad fueron obtenidos por medio del software libre WSxM 5.0 develop 7.0

\subsection{Lagunaridad}

La Lagunaridad es una medida adimensional de la heterogeneidad de un conjunto de datos, con la cual se busca medir el grado de aglomeraciones de los mismos y los padrones espaciales a diferentes escalas. (Mandelbrot-1982)

Entre las aplicaciones para la medida de lagunaridad podemos citar

1. Diagnostico automático de células de tumores (Plotnick R. E.1993, Einstein A. J. 1998)

2. Estudio de ecosistemas (Plotnick R. E.1993)

3. Estudio de morfometría de sólidos porosos.(Armatas G.S.2002)

4. Caracterización de neuronas. (Smith A.J.1996)

5. Caracterización da distribución espacial en células retínales.(Costa L,da F, 2004)

6. Padrones de expresión genética. .(Costa $L$,da F, 2004)

Para las superficies fractales la lagunaridad disminuye con el aumento del tamaño de la caja seleccionada, de acuerdo a la ley de potencia

$$
L(r)=A r^{\alpha}
$$

Donde $\alpha<0$, este valor puede ser calculado de la pendiente de la gráfica $\operatorname{Ln}(\mathrm{L}(r))$ versus $\operatorname{Ln}(r)$

La lagunaridad es definida como la razón entre el segundo momento $\left(Z_{2}\right)$ de la distribución y el cuadrado do primero momento $\left(Z_{1}\right)$

$$
\begin{gathered}
L(r)=\frac{Z_{2}}{\left[Z_{1}\right]^{2}} \\
Z_{2}=\sum_{M} M^{2} \cdot Q(M, r) \\
Z_{1}=\sum_{M} M \cdot Q(M, r) \\
L(r)=\frac{\sum_{M} M^{2} \cdot Q(M, r)}{\left[\sum_{M} M \cdot Q(M, r)\right]^{2}}
\end{gathered}
$$

Donde

$$
n_{r}(i, j)=v-u+1
$$

Es la altura relativa, siendo $i, j$ las coordenadas de la imagen

$$
M_{r}=\sum_{i, j} n_{r}(i, j)
$$

Dada una imagen de una tabla de 4x4 (figura 4), y usando el método Box-Counting Diferencial, que pasa por toda la imagen (figura 5)para cada posición se busca el mayor e menor alturas, por ejemplo la figura $5 a$ se tiene el mayor y menor valor 17 y 8 . Dado un cubo $2 \times 2 \times 2$, la cantidad de cubos necesarios para cubrir todo esos valores máximos y mínimos, está dado por la siguiente relación intensidadldimensión, o sea la cantidad de cajas para $u$ será

$$
\frac{17}{2}=8,5
$$




\begin{tabular}{|c|c|c|c|}
\hline 8 & 17 & 18 & 11 \\
\hline 17 & 12 & 19 & 12 \\
\hline 10 & 9 & 7 & 13 \\
\hline 15 & 19 & 13 & 11 \\
\hline
\end{tabular}

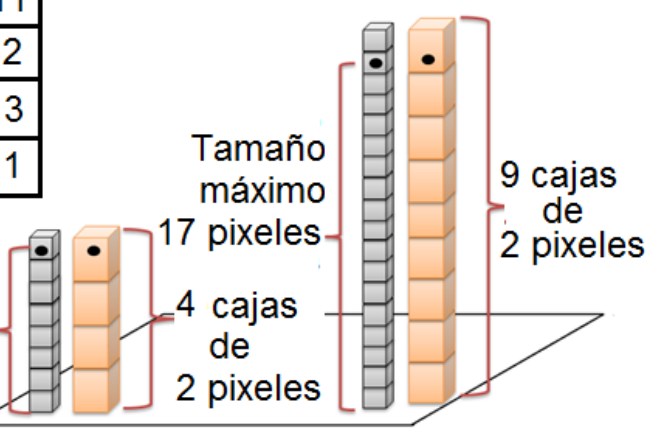

Figura 4: Representación de cajas apiladas

Donde se considera 9 cajas, y para $v$

$$
\frac{8}{4}=4
$$

Entonces tenemos

$$
u-v+1=9-4+1=6
$$

Así se continúa de acuerdo al orden que se presenta

\begin{tabular}{|c|c|c|c|}
\hline 8 & 17 & 18 & 11 \\
\hline 17 & 12 & 19 & 12 \\
\hline 10 & 9 & 7 & 13 \\
\hline 15 & 19 & 13 & 11 \\
\hline
\end{tabular}

(a)

\begin{tabular}{|c|c|c|c|}
\hline 8 & 17 & 18 & 11 \\
\hline 17 & 12 & 19 & 12 \\
\hline 10 & 9 & 7 & 13 \\
\hline 15 & 19 & 13 & 11 \\
\hline
\end{tabular}

(d)

\begin{tabular}{|c|c|c|c|}
\hline 8 & 17 & 18 & 11 \\
\hline 17 & 12 & 19 & 12 \\
\hline 10 & 9 & 7 & 13 \\
\hline 15 & 19 & 13 & 11 \\
\hline
\end{tabular}

(g)

\begin{tabular}{|c|c|c|c|}
\hline 8 & 17 & 18 & 11 \\
\hline 17 & 12 & 19 & 12 \\
\hline 10 & 9 & 7 & 13 \\
\hline 15 & 19 & 13 & 11 \\
\hline
\end{tabular}

(b)

\begin{tabular}{|c|c|c|c||c|c|c|c|}
\hline 8 & 17 & 18 & 11 & 8 & 17 & 18 & 11 \\
\hline 17 & 12 & 19 & 12 & 17 & 12 & 19 & 12 \\
\hline 10 & 9 & 7 & 13 & 10 & 9 & 7 & 13 \\
\hline 15 & 19 & 13 & 11 & 15 & 19 & 13 & 11 \\
\hline
\end{tabular}

\begin{tabular}{|c|c|c|c|c|c|c|c|}
\hline 8 & 17 & 18 & 11 & 8 & 17 & 18 & 11 \\
\hline 17 & 12 & 19 & 12 & 17 & 12 & 19 & 12 \\
\hline 10 & 9 & 7 & 13 & 10 & 9 & 7 & 13 \\
\hline 15 & 19 & 13 & 11 & 15 & 19 & 13 & 11 \\
\hline
\end{tabular}

(e)

Figura 5: Deslizamiento de las cajas usando el método Box-Counting Diferencial

Donde se obtiene la lacunaridad

$$
\mathrm{L}=1,032
$$

Este es el valor del cálculo de la lagunaridad de acuerdo al ejemplo que hemos considerado (cuadro de la figura 4)

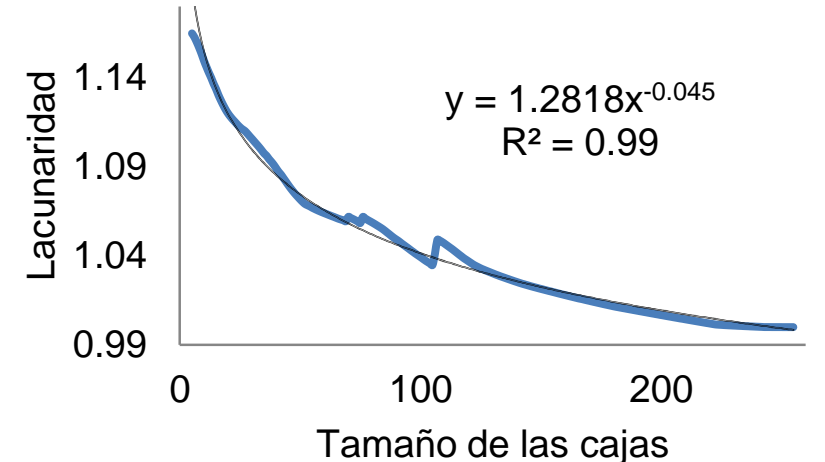

Grafica 2: Lagunaridad de la hoja Copaifera sp.

Esta grafica corresponde a los diferentes valores de lagunaridad respecto al tamaño de las cajas, de la topografía de la Copaifera sp. figura 1, estos valores fueron calculados mediante el programa científico FORTRAN 77, que es un software libre. La lagunaridad es un parámetro fractal que depende de la escala en que se está trabajando, y se rige bajo la ley de potencia, y como la lagunaridad no tiene un valor fijo se obtiene una gráfica de Ln(lagunaridad) versus Ln(tamaño de las cajas), para así poder linealizar la gráfica y obtener un valor constante (pendiente de la recta) para cualquier tamaño de la caja, tal como se muestra en la gráfica 3

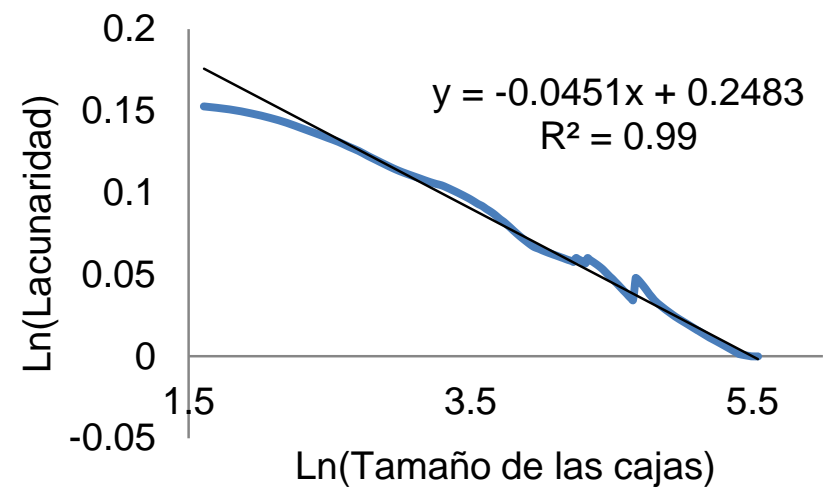

Grafica 3: Linealización de los datos de la hoja de la Copaifera Sp.

\subsection{Succolaridad}

Succolaridad fue definida por Mandelbrot (1977) como un parámetro que informa sobre a conectividad e intercomunicación.

Succolaridad puede ser representado como o grado de penetración de un líquido dentro de un objeto de acuerdo con la dirección de entrada do líquido [5]

\subsubsection{Calculo de la succolaridad}

Se tiene el siguiente cuadro 


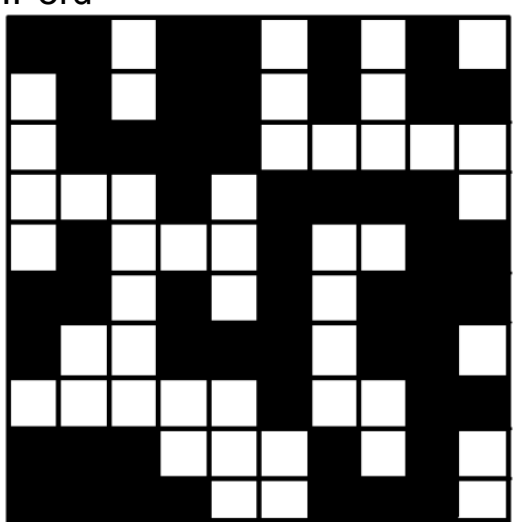

Figura 6: Pixeles negros representan posición vacía o lagunas

Se supone que cada posición del pixel puede ser considerada como vacía (pixel negro) o con masa impenetrable (pixel blanco). Se va a simular la capacidad de drenaje o de percolación de un fluido a través de la imagen. A partir de la imagen a ser analizada (figura 6), se obtienen las cuatro direcciones donde un fluido puede inundar la imagen tal como se muestra en la figura 7

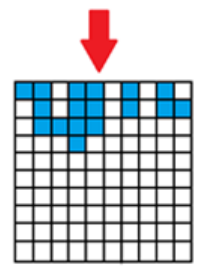

A

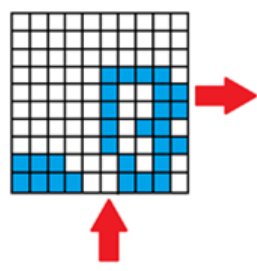

B

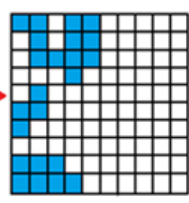

$\mathrm{C}$

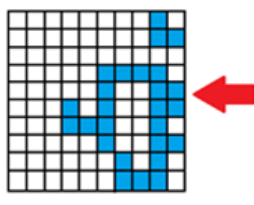

D
Figura 7: Las cuatros direcciones de percolación del fluido

Según Melo para calcular la Succolaridad, cuatro pasos se deben seguir

1.Desde la parte superior de la imagen binaria, todo el borde superior se considera, si el pixel está vacío, significa que el fluido puede pasar y la inundación de esta zona, ya que el pixel está ocupado, la trayectoria se considera un obstáculo para el fluido. Toda la zona se inunda, de forma recursiva, a partir de su vecindario.

2. El siguiente paso consiste en dividir las zonas de inundación en las mismas cajas de tamaño (BS (n), donde $\mathrm{n}$ es el número de posible división de una imagen en cajas) similar al método de conteo de cajas. En la secuencia, el porcentaje de ocupación (OP). Se calcula para cada caja.

3. Para cada cuadro de tamaño $k$, resumir la multiplicación de OP (BS (k)), y $k$ es de 1 a $n$, la presión PR (BS (k), PC), y el PC es la posición ( $x, y)$ del centro de gravedad en el cuadro rango de
Julio 2016

presión aplicada a la caja calculado. La presión varía con el tamaño de la caja tal como se aplica al centroide caja. El centroide caja está dada por $\mathrm{x}$ (Horizontal) e y (vertical).

4. Para el cálculo de la succolaridad tiene la ecuación 6. Según Melo son dos maneras de dividir las figuras, causadas por la presión horizontal y vertical, en las mismas cajas de tamaño, dividiéndolo por un factor $d=9$ (Figura 7A) obteniendo cajas de $1 \times 1$, o por un factor $d=3$, produciendo cajas del tamaño 3x3 (Figura 8).

$$
\begin{gathered}
\delta(B S(k), \text { dir })=\frac{\sum_{k=1}^{n} O P(B S(K)) P R(B S(K), p c)}{\sum_{k=1}^{n} P R(B S(K), p c)} \ldots \\
S_{\downarrow}=\frac{1,7}{20}=0,085 \\
S_{\uparrow}=\frac{7}{20}=0,350 \\
S_{\rightarrow}=\frac{4,3}{20}=0,215 \\
S_{\leftarrow}=\frac{5,8}{20}=0,290
\end{gathered}
$$

Esto son los valores de la succolaridad del cuadro de la figura 6 , mientras más alto sea el valor de la succolaridad, mayor es la penetración del fluido sobre la superficie.

\section{Resultados y discusión}

De acuerdo a la tabla 1, el valor real de la dimensión fractal es $2,1364 \pm 0,0138( \pm 0,65 \%)$, lo cual indica que el área óptima para trabajar es de $25 \times 25 \mu \mathrm{m}^{2}$, La rugosidad va aumentando de acuerdo va aumentando el área de trabajo; pero haciendo un ajuste lineal en dos tramos de los valores de $0,78 \times 0,78$ hasta $12,5 \times 12,5 \mu \mathrm{m}^{2}$ y $25 \times 25$ hasta 100 $\mathrm{x} 100 \mu \mathrm{m}^{2}$ e igualando las ecuaciones lineales se obtiene que el área oprima seria de $25 \times 25 \mu \mathrm{m}^{2}$, con un error de 3,90\%

De acuerdo a la gráfica 2 , se observa que la Lagunaridad de la superficie de la hoja de la Copaifera sp. se rige por la ley de potencia, donde el valor exponencial es $-0,045$, este mismo valor se obtiene en la gráfica 3, donde se hizo un ajuste lineal al graficar Ln (Lagunaridad) versus Ln(tamaño de las cajas) y el valor de la pendiente es -0,0451, este valor es lo que caracteriza a la superficie de la hoja de la Copaifera sp.

La Succolaridad va a depender del sentido del movimiento del fluido a través de la superficie de la hoja, ya que de acuerdo a los valores obtenidos el 
mayor valor se obtuvo cuando el fluido va de abajo hacia arriba $\left(S_{\downarrow}=0,35\right)$, y el menor valor se obtuvo arriba hacia abajo $\left(S_{\downarrow}=0,085\right)$

\section{Conclusiones}

La superficie de la hoja de la Copaifera tiene un comportamiento fractal, y por lo tanto y se rige por la ley de potencia.

La matemática Fractal es una herramienta muy importante para caracterizar superficies, y es muy fácil su manejo.

Los parámetros fractales: Dimensión Fractal, Lagunaridad y Succolaridad se complementa y dan una característica de la superficie.

\section{Agradecimientos}

Mario Calla, agradece a la Universidad Federal de Amapa (UNIFAP), por su apoyo tecnológico y científico.

\section{Referencias}

[1] J. I. R. Cojocaru, D. P. A. I. E. N. "Texture classification based on succolarity. Telecommunications Forum (TELFOR), Belgrade, p. 498-501., 2013.

[2] Hotar, V.; Petr, S. Surface Evaluation by Estimation of Fractal Dimension and Statistical Tools. Hindawi Publishing Corporation, New York, v. 2014, p. 10, August 2014.

[3] Athira Chandra, J. M. Finger Vein Based User Identification Using Differential Box Counting. International Journal of Research in Computer and Communication Technology, Coimbatore, India, v. 3, p. 138-142, January 2014.

Email: mcalla@pucp.pe, jctcarvalho@gmail.com, robert@unifap.br
[4] Barbosa, K. D. S. Distribuição das espécies do gênero Copaifera L. na Amazônia Legal e aspectos morfológicos de C. multijuga Hayne da Reserva de Desenvolvimento Sustentável do Tupé, Manaus-Am. Diversidade Biológica e Sociocultural do Baixo Rio Negro, Manaus, v. 2, p. 135-142, 2009.

[5] Melo, R. H. C.. Using fractal characteristics such as fractal dimension, lacunarity and succolarity to characterize texture patterns on images. Universidade Federal Fluminense. Rio de Janeiro, p. 115. 2007.

[6] Plotnick, R. et al. Lacunarity analysis: A general technique for the analysis of spatial patterns. Physical Review E, Chicago, v. 53, n. 5, maio 1996.

[7] Junior, J. J. et al.. Shape classification using line segment statistics. Elsiever, Brazil, v. 305, p. 349-356, 7 February 2015.

[8] E Rivera et al. Geometria fractal y transformada de Fourier. Scientia et Technica Año XV, v. 48, p. 269-274, Agosto 2011

[9] Coelho, R. C et al.,. The Box Counting Fractal. Dimension: Does it provide an Accurate Subsidy for Experimental Shape Characterization. Anais do VIII SIBGAPI, Sao Paolo, p. 183-190, october 1995.

[10] Ash A. et al. Manual of leaf Architecture:Morphological description and categorization of dicotyledonous and netveined monocotyledonous angiosperms. Leaf Architecture Working Group c/o Scott Wing. , Washington - DC, Estados Unidos, p. 15-44, 1999 